\title{
Mobility based energy efficient and multi- sink algorithms for consumer home networks
}

Article

Accepted Version

Wang, J., Yin, Y., Zhang, J., Lee, S. and Sherratt, R. S. (2013) Mobility based energy efficient and multi-sink algorithms for consumer home networks. IEEE Transactions on Consumer Electronics, 59 (1). pp. 77-84. ISSN 0098-3063 doi: https://doi.org/10.1109/TCE.2013.6490244 Available at https://centaur.reading.ac.uk/32188/

It is advisable to refer to the publisher's version if you intend to cite from the work. See Guidance on citing.

To link to this article DOI: http://dx.doi.org/10.1109/TCE.2013.6490244

Publisher: IEEE

All outputs in CentAUR are protected by Intellectual Property Rights law, including copyright law. Copyright and IPR is retained by the creators or other copyright holders. Terms and conditions for use of this material are defined in the End User Agreement.

www.reading.ac.uk/centaur 
Central Archive at the University of Reading

Reading's research outputs online 


\title{
Mobility based Energy Efficient and Multi-Sink Algorithms for Consumer Home Networks
}

Full Text: published in IEEE Transactions on Consumer Electronics, Volume: 59(1), pp 77 - 84, 2013

Digital Object Identifier: 10.1109/TCE.2013.6490244

Jin Wang, Member, IEEE,

Yue Yin,

Jianwei Zhang,

Sungyoung Lee, Member, IEEE

R. Simon Sherratt, Fellow, IEEE

This work was supported by the National Natural Science Foundation of China (61173072) and the Natural Science Foundation of Jiangsu Province (BK2012461). It was also supported by the industrial Strategic Technology Development Program (10041740) by the MKE Korea, a project funded by Nanjing University of Information Science and Technology (S8110246001) and the National Research Foundation of Korea (NRF) grant funded by the Korea government (MEST) (2011-0030823).

Jin Wang and Yue Yin are with the Jiangsu Engineering Center of Network Monitoring, Nanjing University of Information Science \& Technology, 210044, China (e-mail: \{wangjin, yinyue \} @ nuist.edu.cn).

Jianwei Zhang is with the School of Math and Statistic, Nanjing University of Information Science \& Technology, Nanjing 210044, China (e-mail: zhangjw@nuist.edu.cn).

Sungyoung Lee is with the Computer Engineering Department, Kyung Hee University, Suwon 449-701, Korea (e-mail: sylee@khu.ac.kr).

R. Simon Sherratt is with the School of Systems Engineering, the University of Reading, RG6 6AY, UK (e-mail: sherratt@ieee.org).

\begin{abstract}
With the fast development of the Internet, wireless communications and semiconductor devices, home networking has received significant attention. Consumer products can collect and transmit various types of data in the home environment. Typical consumer sensors are often equipped with tiny, irreplaceable batteries and it therefore of the utmost importance to design energy efficient algorithms to prolong the home network lifetime and reduce devices going to landfill. Sink mobility is an important technique to improve home network performance including energy consumption, lifetime and end-to-end delay. Also, it can largely mitigate the hot spots near the sink node. The selection of optimal moving trajectory for sink node(s) is an NP-hard problem jointly optimizing routing algorithms with the mobile sink moving strategy is a significant and challenging research issue.
\end{abstract}

The influence of multiple static sink nodes on energy consumption under different scale networks is first studied and an Energyefficient Multi-sink Clustering Algorithm (EMCA) is proposed and tested. Then, the influence of mobile sink velocity, position and number on network performance is studied and a Mobile-sink based Energy-efficient Clustering Algorithm (MECA) is proposed. Simulation results validate the performance of the proposed two algorithms which can be deployed in a consumer home network environment.

Index Terms - Wireless Sensor Networks, Sink Mobility, Clustering, Energy Consumption, Network Lifetime. 


\section{INTRODUCTION}

Many kinds of consumer products have been deployed in home automation networks. A home network usually contains a certain type of network connecting many consumer products, e.g. sensors, so that the users can control these appliances in an interactive, intelligent and automatic way to improve their Quality of Life (QoL).

Some representative technologies to implement a home network include: IEEE 802.11, IEEE 1394, Ultra Wide Band (UWB), Bluetooth and ZigBee. ZigBee is highly suitable for implementing home automation networks because various types of sensors can be deployed to collect home data information (e.g. temperature and humidity) in an intelligent and well-organized manner. Wireless Sensor Networks (WSNs) are suitable for the home network environment due to their intrinsic nature including the attributes of self-organizing, infrastructure-less and fault-tolerance. WSNs are usually composed of many sensors and actuators which can sense, process and transmit home raw data to a remote sink node (or BS). Typical home applications of WSNs include home automation, home activity detection (e.g. fall detection, sleeping/living pattern study) and home healthcare [1].

Due to the fact that the home network sensor devices are usually powered by tiny and irreplaceable batteries then it is difficult to recharge them. Thus, the design of energy efficient network layer algorithms is very important to prolong the home network lifetime.

Many energy efficient routing algorithms and protocols have been proposed to prolong network lifetime for WSNs in recent years [2]. In most of these routing scenarios, the sink node is usually assumed to be fixed either inside or outside the WSNs. This will inevitably cause the hot spot phenomenon [3], primarily the sensors close to sink node will have more traffic load to transmit than nodes further away from the sink node under multi-hop transmission. They will consume all their available electrical power much earlier than others, which will cause degraded network performance including network partition, isolated nodes and reduced network lifetime.

By introducing mobility into WSNs, network performance such as energy efficiency, lifetime and end-to-end delay can be vastly improved [4]-[15]. The majority of mobility strategies for WSNs are to deploy mobile relay(s) or sink node(s) rather than mobile sensors, which are easier to implement. Sometimes, the deployment of mobile relays is impractical under harsh environments, whereas the deployment of mobile sinks along the periphery of network is very practical. To achieve mobility, devices including sensors, gateway and PDA can be mounted on mobile devices or people, which is not difficult to realize.

Intuitively, the following advantages can be achieved if the mobile sink nodes are well deployed and scheduled. First, the hot spot problem can be largely mitigated when the sink nodes move around. Second, energy balancing can be achieved among sensor nodes with prolonged network lifetime. Third, transmission latency can be reduced and throughput can be improved under multiple sink nodes environment. Finally, some isolated nodes or data under sparsely deployed networks can be periodically accessed by mobile sink nodes to improve network performance.

As per sink node deployment strategy, it can be categorized into four classes, namely: single static sink, single mobile sink, multiple static sinks and multiple mobile sinks deployment strategy. In this paper, both multiple static sinks and multiple mobile sinks deployment strategies are studied. As is stated in the literature [4], the selection of the optimal moving trajectory for sink nodes is an NP-hard problem. How to jointly optimize the routing algorithms as well as moving strategy of multiple mobile sinks is even a more challenging research issue.

This paper aims to jointly optimize the clustering algorithm and sink node deployment strategy under a smart home network. Two sink mobility based energy-efficient clustering algorithms are first proposed. These two algorithms are termed Energyefficient Multi-sink Clustering Algorithm (EMCA) and Mobile-sink based Energy-efficient Clustering Algorithm (MECA). Then, the influence of multiple sink number, velocity and position on network performance is presented. Since the sink node is more expensive, a mechanism to find the optimal sink number to prevent the overuse of sink nodes is further provided.

The rest of the paper is organized as follows. Section II is a literature survey concerning various sink node deployment strategies for WSNs. Section III gives the home network system model. In Section IV, the EMCA and MECA algorithms are explained in detail. Section V presents extensive simulation results and analysis and Section VI concludes the paper.

\section{RELATED WORKS}

LEACH [3] is one of the most famous hierarchical routing protocols for WSNs, which can guarantee network scalability and prolong network lifetime up to 8 -fold than other ordinary routing protocols. The energy can be well balanced among sensors since each sensor takes turn to become the cluster head at different rounds. However, 5\% of cluster head nodes are randomly chosen and the cluster heads use direct transmission to send their data to the sink node. 
In 2003, Shah et al [5] first proposed the basic idea of mobile sinks for WSNs where the authors call them "Data Mules." The Mules use random walk to pick up data in their close range and then drop off the data to some access points. The energy consumption for sensors can be largely reduced since the transmission range is short.

Younis et al [6] also investigated the potential of base station repositioning to improve network performance. The authors addressed when, where and how the base station should be moved by checking the traffic density of nodes one hop away from base station as well as their relative distance. A Scalable Energy-efficient Asynchronous Dissemination (SEAD) protocol [7] was proposed to minimize energy consumption in both building a dissemination tree and disseminating data to mobile sinks. When the sink joined the tree, the Steiner tree was built recursively and SEAD found the minimal cost entry to the tree for the sink using unicast.

Gandham et al [8] tried to use an ILP (Integer Linear Program) to determine the locations of multiple base stations. They aimed at minimizing the energy consumption per node and prolonging the network longevity.

In 2004 - 2005, the idea of multiple mobile sinks for WSNs was further investigated. Akkaya et al [4] stated that to find the optimal moving positions for mobile sinks was an NP-hard problem in nature. Oyman et al [9] focused on multiple sink location problems and they presented three problems (BSL, MSPOP and MSMNL) depending on design criteria and provided solution techniques. Luo et al [10] formulated lifetime maximization as a min-max problem and jointly studied the sink mobility and routing strategy. They claimed that the overall energy is minimized when the mobile sinks were located at the periphery of the circular network. Wang et al [11] studied the WSNs with one mobile sink and one mobile relay individually and they claimed that the improvement in network lifetime over the all static network was upper bounded by a factor of four. However, more recently, Shi et al [12] proposed theoretical results on the optimal movement of a mobile base station. They showed that when base station location is un-constrained, the network lifetime can be at least $(1-\varepsilon)$ of the maximum network lifetime under their designed joint mobile base station and flow routing algorithm. Marta et al [13] proposed to change mobile sinks' location when the energy of nearby sensors became low. In that case, mobile sinks had to find new zones with richer sensor energy. The authors claimed that an improvement of 4.86 times in network lifetime was achieved compared to the static sink case. Lee et al [14] introduced a single local sink model to minimize total energy cost during geographic routing. The optimal sink location is determined by a global sink and this model was extended to multiple local sinks model to provide scalability. Kim et al [15] proposed an Intelligent Agent-based Routing (IAR) protocol to guarantee efficient data delivery to sink node. Mathematical analysis and experimental results were provided to validate the superiority of their proposed protocol in terms of delay, energy consumption and throughput.

\section{SYSTEM MODEL}

Fig. 1 depicts a home network consisting of various types of sensor nodes such as camera, Micaz, biosensor and RFID, as well as multiple static or mobile sink nodes which play a key role in this paper. The home network is divided into several clusters and there is a Cluster Head $(\mathrm{CH})$ inside each cluster, which can perform data fusion after collecting all the raw data from its ordinary members.

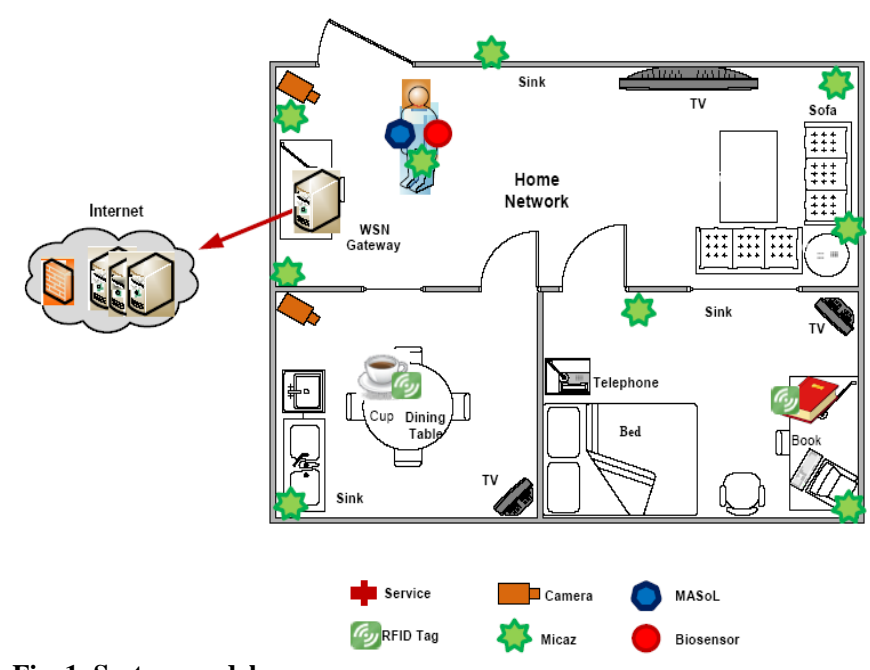

Fig. 1. System model 
Cameras are usually installed in the main room and dining room, which is not suitable for the living room. Biosensors are attached to the body to collect human physical information. Sink nodes can be installed either on the wall (fixed) or attached on the body (mobile) to collect raw data from various sensor nodes. There is a home gateway server which will communicate with both the inside and outside devices via a wired or wireless communication. For example, it will receive commands from users and delivery requests to certain sensor or sink nodes.

The main purpose of this paper is to study the influence of fixed and mobile sink strategies on home network performance in terms of energy consumption, network lifetime as well as to mitigate the hot spot problem. When fixed sink nodes are deployed, the home network is divided into several clusters and the optimal sink number is studied. When mobile sink nodes are deployed (e.g. on human body), the influence of sink moving velocity, position and number of sink nodes on home network performance is studied.

A traditional home network can be modeled as a graph $G(N, E)$ where $N$ is the set of all sensor nodes and $E$ is the set of all links $(i, j)$. Here, $i$ and $j$ are neighboring nodes. Node $i$ can communicate directly with its neighbor node $j$ if their Euclidean distance is smaller than its transmission radius.

Here, the first order radio model [2], [3] is used as the energy model. Based on the distance between transmitter and receiver, a free space ( $d^{2}$ power loss) or multi-path fading ( $d^{4}$ power loss) channel models are used. Each sensor node consumes $E_{T x}$ amount of energy to transmit a $l$ bits packet over distance $d$ and $E_{R x}$ for reception, where $E_{\text {elec }}$ is the energy dissipated on circuit, and $\varepsilon_{f s}$ and $\varepsilon_{m p}$ are free space and multi-path fading channel parameters respectively.

$$
\begin{aligned}
& \mathrm{E}_{\mathrm{Tx}}(l, d)=\left\{\begin{array}{l}
l E_{\text {elec }}+l \varepsilon_{f s} d^{2}, d<d_{0} \\
l E_{\text {elec }}+l \varepsilon_{m p} d^{4}, d \geq d_{0}
\end{array}\right. \\
& E_{R x}(l)=l E_{\text {elec }}
\end{aligned}
$$

The following assumptions regarding the system model are made:

- Wireless links are bi-directional and symmetric.

- $\quad$ Sensors are homogeneous and stationary after deployment.

- $\quad$ Sink nodes are energy unconstraint and they can move freely.

- Ideal MAC layer with no collisions is supported.

- $\quad$ Sensors can adjust their power based on the relative distance.

\section{The Proposed EMCA ANd MECA Algorithms}

In this section, the influence of multiple static and mobile sink nodes on network performance is studied under different scale hierarchical networks. Two sink mobility based energy efficient clustering algorithms for WSNs are proposed, namely an Energy-efficient Multi-sink Clustering Algorithm (EMCA) as well as a Mobile-sink based Energy-efficient Clustering Algorithm (MECA).

\section{A. Energy-efficient Multi-sink Clustering Algorithm (EMCA)}

The entire network is divided into several clusters, as depicted in Fig. 2. In each cluster, there is one Cluster Head $(\mathrm{CH})$ for data collection and the rest of the sensors are called ordinary nodes. The $\mathrm{CH}$ is determined by the residual energy among sensors and the $\mathrm{CH}$ sends aggregated data to the relevant sink. By adopting clustering or hierarchical routing technique, network scalability and easier management can be guaranteed. If the clustering algorithm is well designed with CHs located in a geographically more uniform way, energy consumption can be well balanced and reduced, causing a much prolonged network lifetime. 


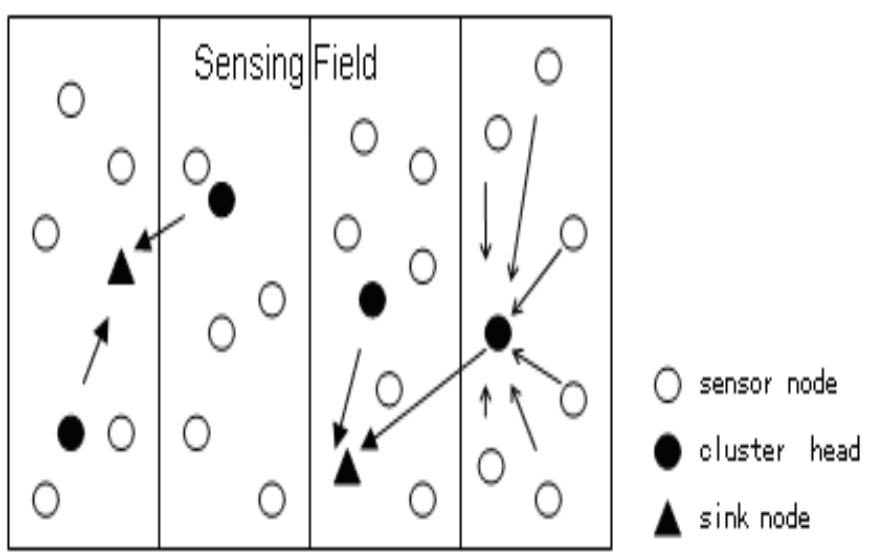

Fig. 2. Cluster formation in EMCA

In EMCA, each cluster head selects an optimal sink to send aggregated data. The reduction and the balancing of the energy consumption is the primary concern. For any $\mathrm{CH}_{n}$, the energy consumption to sink node $B S_{k}$ is represented as:

$\mathrm{E}\left(C H_{n}, B S_{k}\right)=\left\{\begin{array}{l}l E_{\text {elec }}+l \varepsilon_{f s} d\left(C H_{n}, B S_{k}\right)^{2}, d<d_{0} \\ l E_{\text {elec }}+l \varepsilon_{f s} d\left(C H_{n}, B S_{k}\right)^{2}, d \geq d_{0}\end{array}\right.$

Equ. (3) shows that the smaller $d\left(\mathrm{CH}_{n}, B S_{k}\right)$ is, the smaller $\mathrm{E}\left(\mathrm{CH}_{\mathrm{n}}, \mathrm{BS}_{\mathrm{k}}\right)$ will be. Inter-cluster algorithm can be formulated as to find the $\operatorname{Min}\left(d\left(\mathrm{CH}_{n}, B S_{k}\right)\right)$.

In many clustering algorithms, such as LEACH, some sensor nodes in the same cluster send data directly to the cluster head. Due to the fact of various locations, certain sensor nodes may consume large amount of energy based on long-distance transmission. Therefore, multi-hop routing is used here. For any member node $S_{i}$ in a cluster, the energy consumption to send data to its $\mathrm{CH}_{S_{i}}$ is represented as:

$\mathrm{E}_{1}\left(S_{i}, C H_{S_{i}}\right)=\left\{\begin{array}{l}1 \mathrm{E}_{\text {elec }}+l \varepsilon_{f s} d\left(S_{i}, C H_{S_{i}}\right)^{2}, d<d_{0} \\ 1 \mathrm{E}_{\text {elec }}+l \varepsilon_{f s} d\left(S_{i}, C H_{S_{i}}\right)^{2}, d \geq d_{0}\end{array}\right.$

In the mean time, $S_{i}$ tries to find another sensor node $S_{\mathrm{j}}$ to relay data to save energy by avoiding directly communication with $\mathrm{CH}_{S_{i}}$. To deliver a $l$-length packet to the cluster head, the energy consumption $\mathrm{E}_{2}\left(S_{i}, S_{j}, C H_{S_{i}}\right)$ is calculated as (5) and the optimal relay node is determined based on the smallest value of $\mathrm{E}_{2}\left(S_{i}, S_{j}, \mathrm{CH}_{S_{i}}\right)$.

$$
\begin{aligned}
& \mathrm{E}_{2}\left(S_{i}, S_{j}, C H_{S_{i}}\right)=E_{T x}\left(l, d\left(s_{i}, s_{j}\right)\right) \\
& +E_{R x}(l)+E_{T x}\left(l, d\left(s_{i}, C H_{S_{i}}\right)\right)
\end{aligned}
$$

As the multiple sink nodes are randomly deployed then in practice some nodes may consume less energy through sending data directly to the sink rather than to its cluster head. 


\section{B. Mobile-sink based Energy-efficient Clustering Algorithm (MECA)}

1) Relocation of sink nodes

In MECA, the moving velocity $v$ of the sink is pre-determined. A sink node only needs to broadcast across the network to inform all sensor nodes of its current location $P_{0}$ at the very beginning for just one time. Later on, as sensor nodes keep record of the original location of the sink, they can reduce the changed angle $\theta$ after a time interval $\Delta \mathrm{t}$.

$v=\frac{\theta^{*} R}{\Delta t} \Rightarrow \theta=\frac{v^{*} \Delta t}{R}$

As $P_{0}$ is known, the new location $P_{\Delta t}$ can be determined, as is shown in Fig. 3.

After the broadcasting finishes, the mobile sink is ready to collect data. Here, the mobile sink is assumed to stay at a site for a period long enough to complete a round of data collection, and then moves to the next position.

2) Cluster formation and cluster head selection

As depicted in Fig. 4, the whole sensor network is divided into several clusters. When the $\mathrm{CH}$ selection begins, the sensor node that is located in the center of each cluster is motivated, like $S_{i}$ and is regarded as the $\mathrm{CH}$ candidate. It broadcasts one message within a neighborhood of radius $R$. This message aims to motivate other nodes for the competition of the cluster head. It contains the node's id and its residual energy. Only the nodes within the transmission range can receive the message and become active, whereas the outside nodes remain idle. If any node $S_{\mathrm{j}}$ has larger residual energy than $S_{i}$, it becomes the new cluster head candidate and broadcasts new message with its own information to the others. If $S_{\mathrm{j}}$ has equal residual energy with $S_{i}$, compare the ID. The node with a smaller ID wins. If $S_{\mathrm{j}}$ has smaller residual energy than $S_{i}$, it still broadcasts the message of $S_{i}$ . As soon as the comparison is done, the unselected node becomes idle again. All nodes in the cluster should be compared only once. In this way, the node with the largest residual energy is chosen as the cluster head.

\section{3) Hierarchical routing phase}

For node $S_{i}$ in one cluster, the energy consumption cost to send data to its cluster head $\mathrm{CH}_{S_{i}}$ is given in Equ. (4). In the mean time, $S_{i}$ tries to find another $S_{\mathrm{j}}$ to relay data which may consume less energy than that through directly communication with $\mathrm{CH}_{S_{i}}$. Since the direction of data transmission can be randomly chosen, various nodes can be chosen, which turn out to cause various energy consumption.

Suppose $S_{i}$ chooses $S_{\mathrm{j}}$ as its relay node and let $S_{\mathrm{j}}$ have direct communication with the $C H_{S_{i}}$. To deliver a $l$-length packet to the $\mathrm{CH}$, the energy consumed by $S_{i}$ and $S_{\mathrm{j}}$ is shown in (5).

Each $S_{i}$ chooses $S_{\mathrm{j}}$ with the smallest $\mathrm{E}_{2}\left(S_{i}, S_{j}, \mathrm{CH}_{S_{i}}\right)$ as the relay node if necessary:

$\mathrm{E}_{2}\left(S_{i}, C H_{S_{i}}\right)=\operatorname{Min}\left(E_{2}\left(S_{i}, S_{j}, C H_{S_{i}}\right)\right)$

Compare (4) and (7), and the smaller one is chosen:

$$
E\left(S_{i}, C H_{S_{i}}\right)=\operatorname{Min}\left(E_{1}\left(S_{i}, C H_{S_{i}}\right), E_{2}\left(S_{i}, C H_{S_{i}}\right)\right.
$$

In MECA, the sink node changes its location over time. Therefore, some nodes may consume less energy through sending data directly to the sink rather than to its cluster head. So it is necessary to compare $E\left(S_{i}, C H_{S_{i}}\right)$ and $E\left(S_{i}, B S\right)$ and decide the final route. In summary, the clustering algorithms in this paper can be viewed as to find the $\operatorname{Min}\left(E\left(S_{i}, C H_{S_{i}}\right), E\left(S_{i}, B S\right)\right)$. 


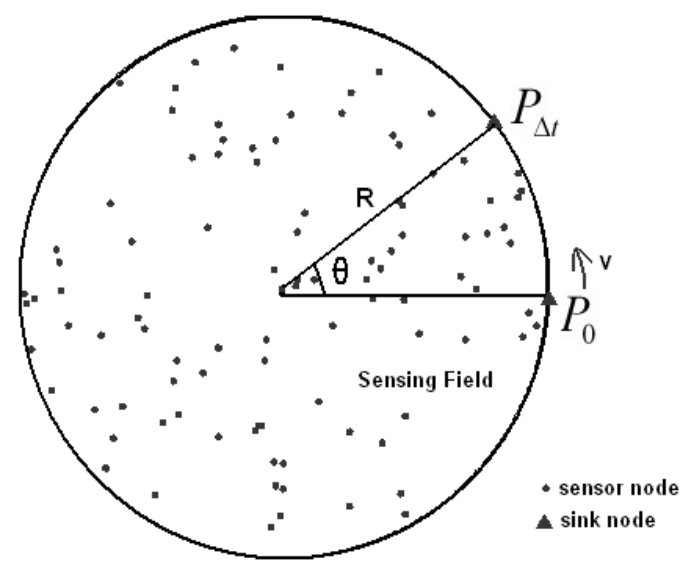

Fig. 3. Relocation of sink nodes in MECA

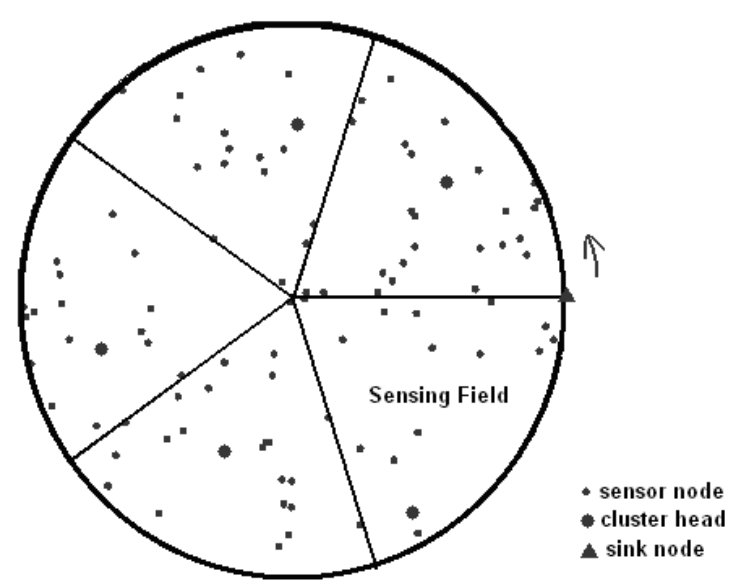

Fig. 4. Cluster formation in MECA

\section{Performance Evaluation}

\section{A. Test Environment}

As is shown in Table I, there are 100 sensor nodes deployed in a [300,300] to [500, 500] network with multiple sink nodes placed either inside or along the periphery of the area. The maximum transmission radius is assumed to be 120 meters to cover even the most demanding home network. Each node takes turn to transmit a 6-bit message to their nearby sink node using either direct transmission or multi-hop transmission based on different routing algorithms. The two algorithms are compared with other popular routing algorithm like LEACH [3] in terms of energy consumption and network lifetime.

TABLE I

NETWORK PARAMETERS

\begin{tabular}{ll}
\hline \hline Parameter name & Value \\
\hline Network size & {$[300,300]$ to $[500,500]$} \\
Num. of nodes (N) & 100 \\
Radius (R) & $120 \mathrm{~m}$ \\
Packet length (1) & $6 \mathrm{bits}$ \\
Initial energy (E $\left.\mathrm{E}_{0}\right)$ & $0.5 \mathrm{Joul}$ \\
Energy consumption on circuit & $50 \mathrm{~nJ} / \mathrm{bit}$ \\
Free-space channel parameter & $10 \mathrm{pJ} / \mathrm{bit} / \mathrm{m}^{2}$ \\
Multi-path channel parameter & $0.0013 \mathrm{pJ} / \mathrm{bit} / \mathrm{m}^{4}$ \\
\hline
\end{tabular}




\section{B. EMCA Performance Analysis}

Fig. 5 illustrates the total energy consumption in joule unit of EMCA under different scale networks. The whole network is divided into several clusters and the multiple static sink nodes are randomly deployed in the network. It can be seen that the total energy consumption units decreases as the number of sinks increases. When 3 or 4 sinks are deployed, the decreasing rate of energy consumption becomes relatively small even if more sink nodes are added later.

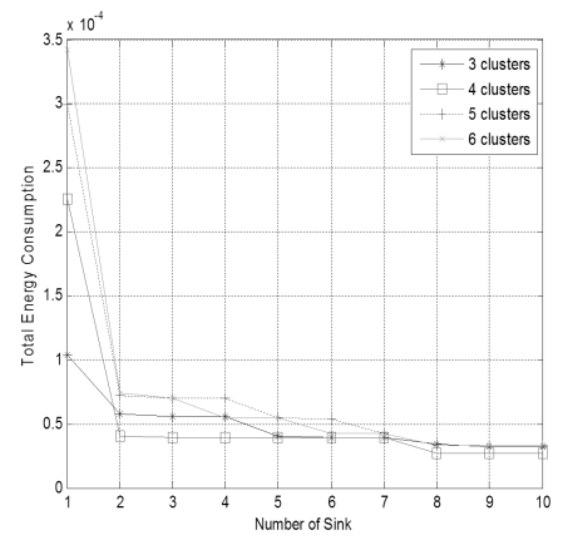

(a) Total energy consumption in $[300,300]$ network

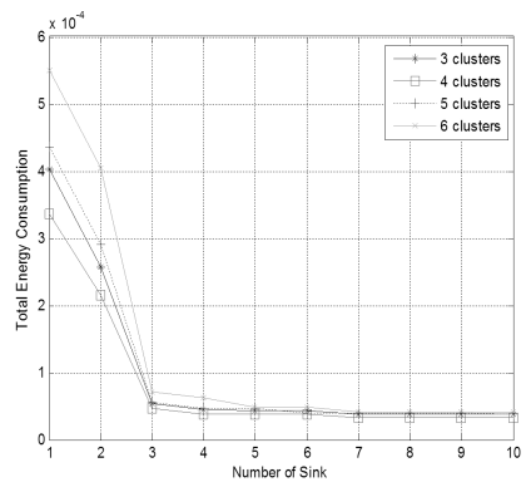

(b) Total energy consumption in $[500,500]$ network

Fig. 5. Total energy consumption under different scale networks

Even though the introduction of more sink nodes can save more energy, the cost of sink $\mathrm{C}_{\text {sink }}$ is usually much higher than the cost of sensor node $\mathrm{C}_{\text {sensor }}$. Thus, the optimal sink number under different scale networks needs to be found. Here, a metric termed $\mathrm{E} \times \mathrm{C}$ is defined, where $\mathrm{E}$ is the energy consumption and $\mathrm{C}$ is set as $\left(1+\frac{\mathrm{C}_{\text {sink }}}{\mathrm{N}^{*} \mathrm{C}_{\text {sensor }}} \cdot k\right)$ with $N$ sensors and $k$ sinks. Fig. 6 depicts the $\mathrm{E} \times \mathrm{C}$ performance with different sink number under $500 \times 500 \mathrm{~m}^{2}$ network. It can be seen that 3-8 sinks can provide relatively small value of $\mathrm{E} \times \mathrm{C}$. As the cost ratio between sink and sensor becomes larger (e.g. 50:1), it is clear that the deployment of 3 sink nodes under such network has the best performance. 


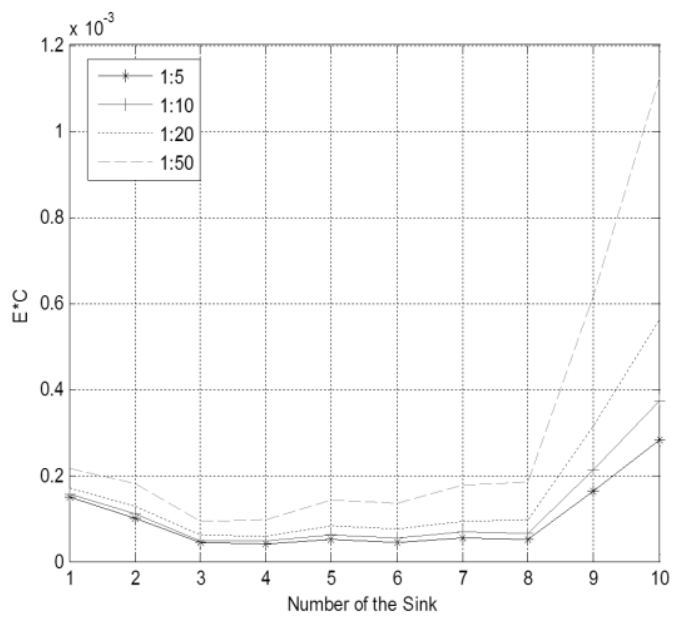

Fig. 6. Optimal sink number in EMCA

The total energy consumption of EMCA and LEACH in joule unit under $500 \times 500 \mathrm{~m}^{2}$ network is also studied, as depicted in Fig. 7. In 20 rounds, EMCA remains much better performance with less energy consumption than LEACH. This is mainly because of the clustering method that distributes CHs more evenly in EMCA to reduce transmission cost along the path. Also, the multi-hop transmission can save energy inside each cluster.

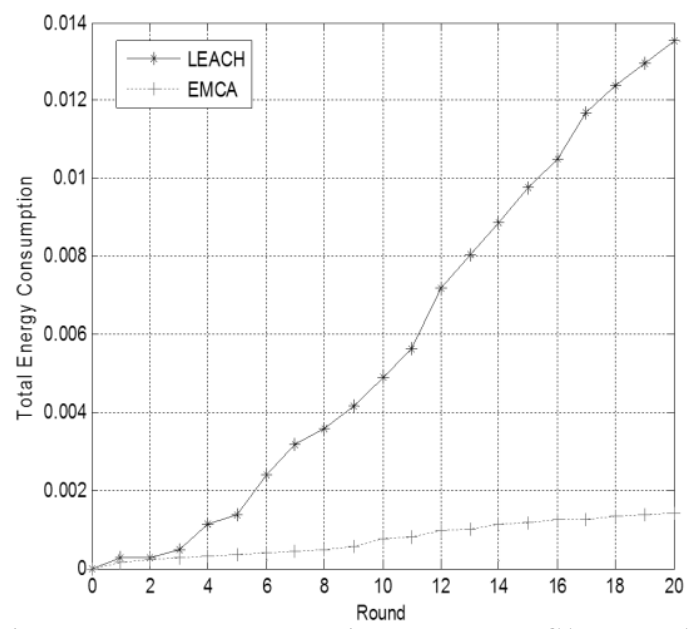

Fig. 7. Total energy consumption between EMCA and LEACH

\section{MECA Performance Analysis}

The influence of single mobile sink moving strategy on energy consumption is first studied. As depicted in Fig. 8, there are 100 nodes randomly deployed in a $\mathrm{R}=500 \mathrm{~m}$ circular network. The single sink node can either move with different angular velocity $(20,30,60$ angles/s), or move along the periphery of each circle with different radius (1/5R, 2/5R, $R)$. 


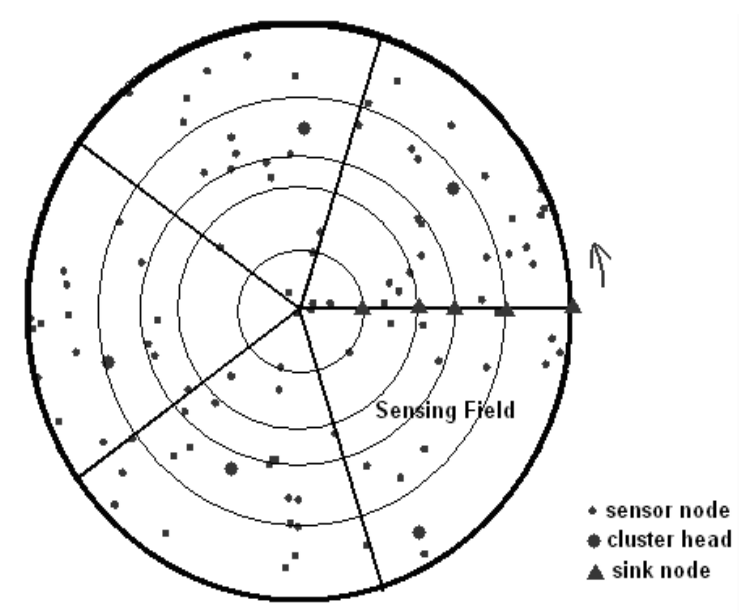

Fig. 8. Single mobile sink moving strategy in MECA

It can be seen from Fig. 9 that a single mobile sink velocity and position have little influence on energy consumption of sensor nodes due to the average distance square being similar to the single moving sink regarding the random sensor network topology.

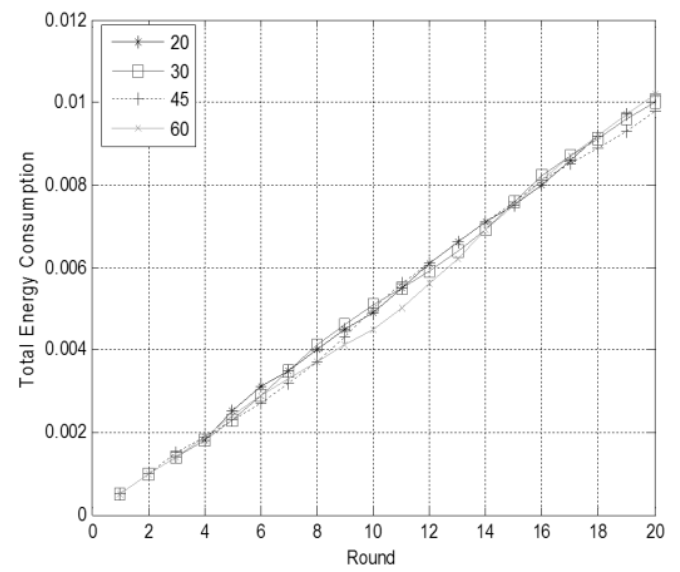

(a) Sink moving velocity influence

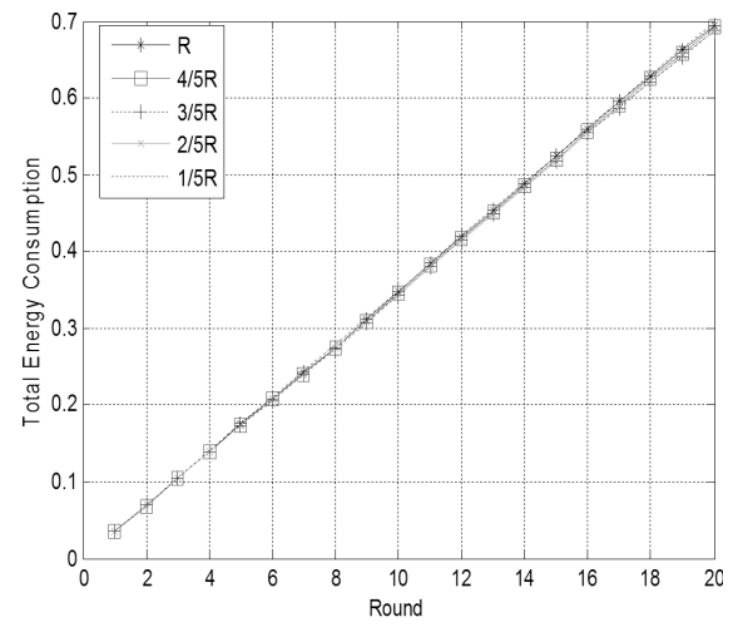

(b) Sink moving position influence

Fig. 9. Influence of sink moving strategy on energy consumption

In Fig. 10, the influence of multiple mobile sink nodes on energy consumption is shown under $\mathrm{R}=300 \mathrm{~m}$ and $\mathrm{R}=500 \mathrm{~m}$ circular networks. The number of mobile sinks varies from one to five along the periphery of network. 


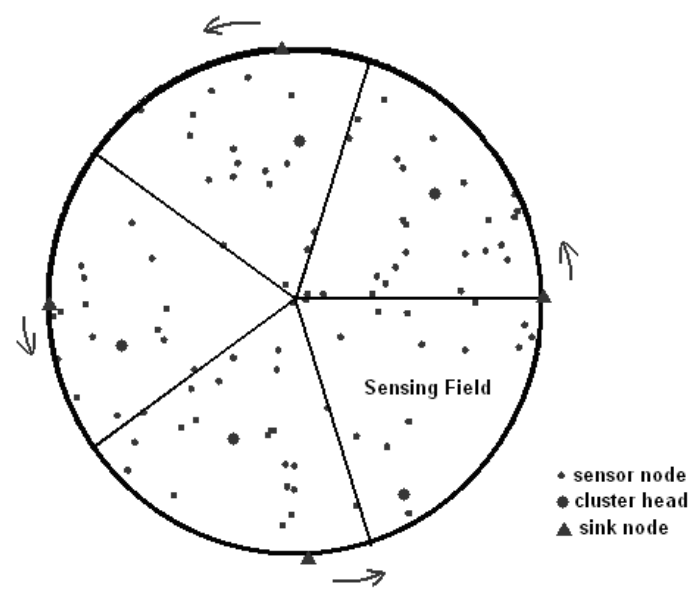

Fig. 10. Multiple mobile sinks in MECA

It can be seen from Fig. 11 that total energy consumption decreases as the number of the sinks increases. If four mobile sinks are deployed, the total energy consumption becomes small enough that introduction of more sinks will hardly make any reduction of energy consumption. Thus it is concluded that four sink nodes are actually enough regarding $R=300 \mathrm{~m}$ and $\mathrm{R}=500 \mathrm{~m}$ networks. This is similar to the conclusion of Fig. 6 for EMCA. Finally, the influence of multiple mobile sink nodes on network lifetime is studied under $\mathrm{R}=300 \mathrm{~m}$ circular network, where four mobile sink nodes are used.

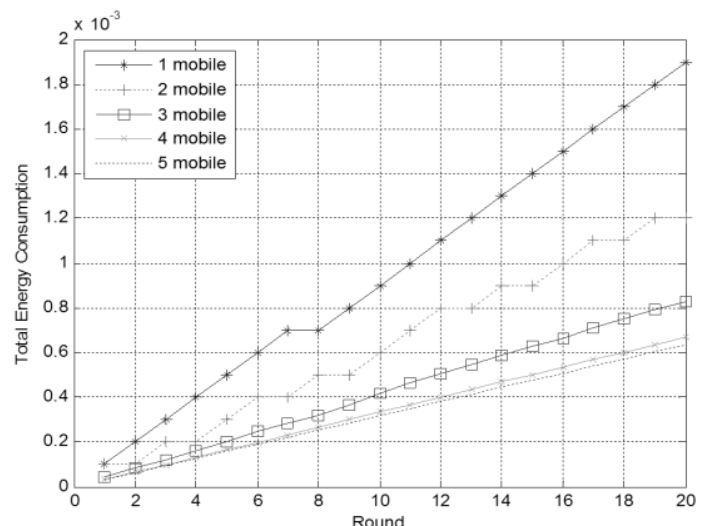

(a) Total energy consumption in $\mathrm{R}=300 \mathrm{~m}$ network

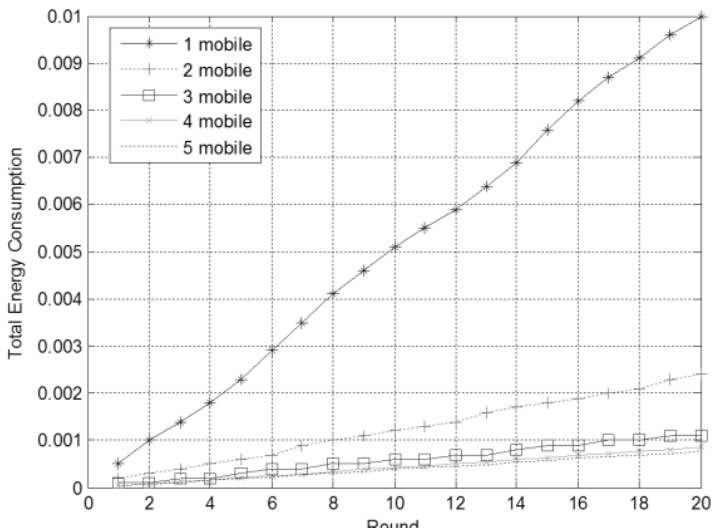

(b) Total energy consumption in $\mathrm{R}=500 \mathrm{~m}$ network

Fig. 11. Influence of mobile sink number on energy consumption

From Fig. 12, it can be seen that the MECA has much better performance than LEACH in terms of number of nodes alive and 
average of residual energy. If network lifetime is defined as the time when the first node dies out of energy, the lifetime of MECA is two times (1800 rounds) longer than LEACH (600 rounds) in Fig. 12(a). In Fig. 12(b), the average residual energy of LEACH also decreases more sharply than MECA, which means LEACH consumes more average energy than MECA during routing process.

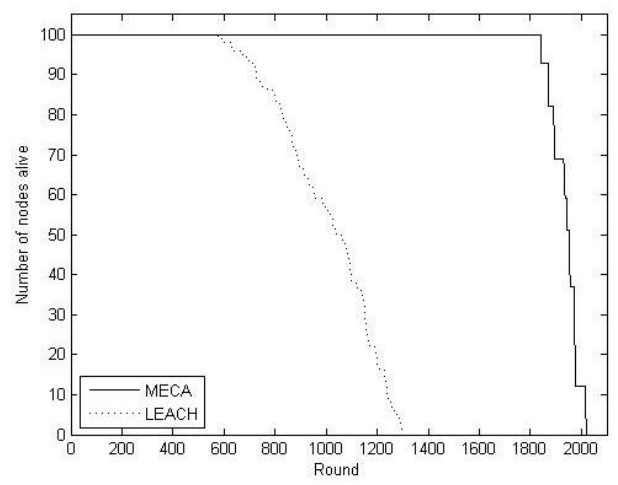

(a) Number of nodes alive

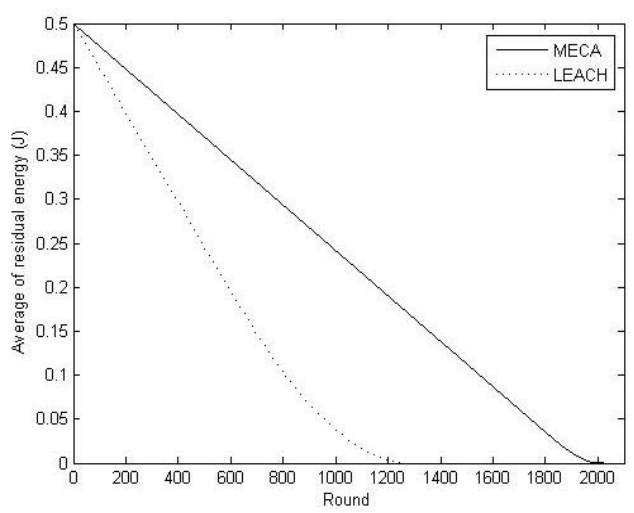

(b) Average of residual energy

Fig. 12. Influence of multiple mobile sink on network lifetime

\section{CONCLUSION}

Sink mobility has a very significant impact on the network performance, especially for a smart home network or networks without a fixed infrastructure. In this paper, a survey on relevant multiple static and mobile sinks strategies for WSNs is first presented. Then, two sink mobility based energy efficient clustering algorithms are proposed for WSNs, namely EMCA and MECA. The influence of multiple sink number, velocity and position on energy consumption and network lifetime was carefully studied through extensive simulation and analysis. A cost function of sink node is also provided to find the optimal multiple sink number as per different scale WSNs.

In a home network environment, multiple static and mobile sinks based energy efficient routing algorithms can be applied in typical consumer products, such as sensors, which can coordinate with each other in a more intelligent and well-organized manner.

\section{REFERENCES}

[1] I. Akyildiz, W. Su, Y. Sankarasubramaniam, E. Cayirci, "Wireless sensor networks: a survey,” Journal of Computer Networks, vol. 38, no. 4, pp. 393-422, March 2002.

[2] K. Akkaya, M. Younis, “A survey on routing protocols in wireless sensor networks," Elsevier Ad Hoc Network Journal, vol. 3, no. 3, pp. 325-349, May 2005.

[3] W. R. Heinzelman, A. Chandrakasan, H. Balakrishnan, "Energy-efficient communication protocol for wireless microsensor networks," Proc. of the 33rd Annual Hawaii International Conference on System Sciences (HICSS), pp. 1-10, Jan. 2000.

[4] K. Akkaya, M. Younis, M. Bangad, "Sink repositioning for enhanced performance in wireless sensor networks," Elsevier Computer Networks, vol. 49, no. 4, pp. 512-534, Nov. 2005. 
[5] R. C. Shah, S. Roy, S. Jain, W. Brunette, "Data MULEs: modeling a three-tier architecture for sparse sensor networks," Proc. of the 1st IEEE International Workshop on Sensor Network Protocols and Applications, pp. 30-41, May 2003.

[6] M. Younis, M. Bangad, K. Akkaya, "Base-station repositioning for optimized performance of sensor networks," Proc. of the Vehicular Technology Conference, vol. 5, pp. 2956-2960, Oct. 2003.

[7] H. S. Kim, T. F. Abdelzaher, W. H. Kwon, "Minimum-energy asynchronous dissemination to mobile sinks in wireless sensor networks," Proc. of the 1st International Conference on Embedded Networked Sensor Systems, pp. 193-204, Nov. 2003.

[8] S. R. Gandham, M. Dawande, R. Prakash, S. Venkatesan, "Energy efficient schemes for wireless sensor networks with multiple mobile base stations," Proc. of the Global Telecommunications Conference, vol.1, pp. 377-381, Dec. 2003.

[9] E. I. Oyman, C. Ersoy, "Multiple sink network design problem in large scale wireless networks," Proc. of IEEE International Conference on Communications, vol.6, pp. 3663-3667, June 2004.

[10] J. Luo, P. Hubaux, "Joint mobility and routing for lifetime elongation in wireless sensor networks," Proc. of the 24th Annual Joint Conference of the IEEE Computer and Communications Societies, vol. 3, pp. 1735-1746, Mar. 2005.

[11] W. Wang, V. Srinivasan, K. Chua, "Using mobile relays to prolong the lifetime of wireless sensor networks", Proc. of the 11th annual international conference on Mobile Computing and Networking, pp. 270-283, Aug. 2005.

[12] Y. Shi, Y. T. Hou, "Theoretical results on base station movement problem for sensor network," Proc. of the 27th Conference on Computer Communications, pp. 1-5, April 2009.

[13] M. Marta, M. Cardei, "Improved sensor network lifetime with multiple mobile sinks", Elsevier Pervasive and Mobile Computing, vol. 5, no. 5, pp. 542555, Oct. 2009.

[14] E. Lee, S. Park, F. Yu, S. H. Kim, "Data gathering mechanism with local sink in geographic routing for wireless sensor networks", IEEE Trans. Consum. Electron., vol. 56, no. 3, pp. 1433-1441, Aug. 2010.

[15] J. W. Kim, J. S. In, K. Hur, J. W. Kim, D. S. Eom, "An intelligent agent-based routing structure for mobile sinks in WSNs", IEEE Trans. Consum. Electron., vol. 56, no. 4, pp. 2310-2316, Nov. 2010.

\section{BIOGRAPHIES}

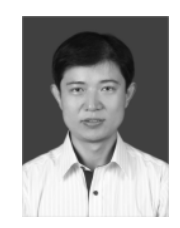

Jin Wang (M'11) received the B.S. and M.S. degree in the Electronical Engineering from Nanjing University of Posts and Telecommunications, China in 2002 and 2005, respectively. He received Ph.D. degree in the Ubiquitous Computing laboratory from the Computer Engineering Department of Kyung Hee University Korea in 2010. Now, he is a professor in the Computer and Software Institute, Nanjing University of Information Science and technology. He has published more than 70 journal and conference papers. His research interests mainly include routing protocol and algorithm design, performance evaluation and optimization for wireless ad hoc and sensor networks.

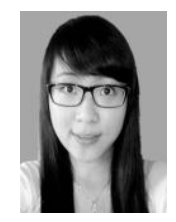

Yue Yin received the bachelor degree in Applied Computing major from the Institute of Computer and Software, Nanjing University of Information Science and Technology in 2011. She is currently pursuing the master degree in the same Institute with Technology of Computer Application major. Her research interests include routing algorithms and data replication for wireless sensor networks.

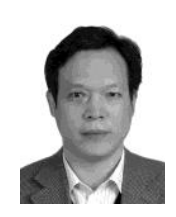

Jianwei Zhang received the B.S. degree with mathematics major from Suzhou University in 1986, M.S. degree with computer application major from Wuhan University in 1998, and the $\mathrm{Ph}$. D. degree with pattern recognition and intelligent system major from Nanjing University of Science and Technology in 2006. He is now a professor in Nanjing University of Information Science \& Technology. His main research interests include image processing, pattern recognition and numerical analysis.

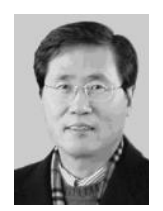

Sungyoung Lee (M’05) received his B.S. from Korea University, Seoul, Korea. He got his M.S. and Ph.D. degrees in Computer Science from Illinois Institute of Technology (IIT), Chicago, USA in 1987 and 1991 respectively. He has been a professor in the department of Computer Engineering, Kyung Hee University, Korea since 1993. He is a founding director of the Ubiquitous Computing Laboratory, and has been affiliated with a director of Neo Medical ubiquitous-Life Care Information Technology Research Center, Kyung Hee University since 2006. Before joining Kyung Hee University, he was an assistant professor in the Department of Computer Science, Governors State University, Illinois, USA from 1992 to 1993. His current research focuses on Ubiquitous Computing and Applications, Wireless Ad-hoc and Sensor Networks, Context-aware Middleware, Sensor Operating Systems, Real-Time Systems and Embedded Systems.

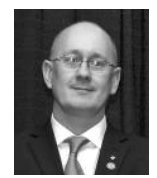

R. Simon Sherratt (M'97-SM'02-F'12) received the B.Eng. degree in Electronic Systems and Control Engineering from Sheffield City Polytechnic, UK in 1992, M.Sc. in Data Telecommunications in 1994 and Ph.D. in video signal processing in 1996 both from the University of Salford. Since 1996, he has been a Lecturer in Electronic Engineering at the University of Reading, currently a Senior Lecturer in Consumer Electronics and a Director for Teaching and Learning. His research topic is signal processing in consumer electronic devices concentrating on equalization and DSP architectures.

Eur Ing Dr. Sherratt has served the IEEE Consumer Electronics Society as a Vice President (Conferences) (2008/9), AdCom member (20032008, 2010-2012) and Awards chair (2006/7). He is a member of the IEEE Transactions on Consumer Electronics Editorial Board (2004-) and currently the Editor-in-Chief (2011-), also the IEEE International Conference on Consumer Electronics general chair 2009, IEEE International Symposium on Consumer Electronics general chair 2004. He received the IEEE Chester Sall 1st place best Transactions on Consumer Electronics paper award in 2004 and the best paper in the IEEE International Symposium on Consumer Electronics in 2006. 